\title{
Descripción de un caso de mieloencefalitis equina por protozoos (EPM) en Argentina
}

\author{
Case report of equine protozoal myeloencephalitis (EPM) in Argentina
}

\author{
Moré Gastón (i) 1,2,*, Monina Marta ${ }^{3}$, Girotti Gabriela ${ }^{3}$, Idiart Julio 4 , \\ Venturini LuCila ${ }^{3}$, Venturini María Cecilia ${ }^{1}$
}

1. Laboratorio de Inmunoparasitología (LAINPA), Facultad de Ciencias Veterinarias, Universidad Nacional de La Plata. Calles 60 y 118, La Plata, Argentina.

2. Consejo Nacional de Investigaciones Científicas y Técnicas (CONICET), CCT - La Plata, provincia de Buenos Aires, Argentina.

3. Profesional independiente, provincia de Buenos Aires, Argentina.

4. Profesor emérito, Facultad de Ciencias Veterinarias, Universidad Nacional de La Plata. Calles 60 y 118, La Plata, Argentina.

* Correo electrónico de la autora de contacto: gastonmore@fcv.unlp.edu.ar

\begin{abstract}
Resumen
La mieloencefalitis equina por protozoos (EPM) es causada por Sarcocystis neurona y genera importantes pérdidas económicas. En Argentina, se han detectado anticuerpos contra este protozoo, pero no se han reportado casos clínicos. Una yegua de 12 años, de la provincia de Buenos Aires presentó marcha asincrónica y arpeo bilateral. El animal fue tratado con fenitoína, vitamina B1, AINEs y tenectomía. El cuadro clínico fue progresivo con depresión, parálisis facial, atrofia de los músculos del dorso, lomo y miembros pelvianos, dismetría e inestabilidad de la marcha, temblores generalizados y tetraparesia, por lo que se decidió la eutanasia. Se observaron lesiones microscópicas en el tálamo, el metencéfalo y la médula espinal, y consistieron en pequeños focos múltiples hemorrágicos y focos de inflamación crónica. La inmunohistoquímica realizada en muestras de cerebro y médula espinal reveló la presencia de merontes y merozoitos de $S$. neurona. Mediante PCR-RFLP se observó un patrón de restricción específico de $S$. neurona en muestras de cerebro. La detección de anticuerpos contra $S$. neurona en caballos, la abundancia de comadrejas y la confirmación específica en este caso, sugieren que la enfermedad puede estar extendida en nuestro medio. Esta es la primera descripción de un caso de EPM en Argentina, confirmado mediante estudios histopatológicos, inmunohistoquímicos y moleculares.
\end{abstract}

\section{Palabras clave}

Sarcocystis neurona, EPM, diagnosis, Argentina

\begin{abstract}
Equine protozoal myeloencephalitis (EPM) is mainly caused by Sarcocystis neurona and generates significant economic losses. In Argentina, antibodies to this protozoan have been detected but no clinical cases have been reported. A 12-year-old mare, from Buenos Aires Province, presented asynchronous walking and bilateral stringhalt. The animal was treated with phenytoin, vitamin B1, non-steroid antiinflammatory drugs and subsequent tenectomy. Clinical outcome progressed to depression, facial paralysis, atrophy of the muscles of the back, loin and pelvic limbs, dysmetria and gait instability, generalized tremors and tetraparesis. Euthanasia was decided based on clinical progression and lack of response to treatment. Microscopic lesions were observed in thalamus, metencephalon and spinal cord, and consisted in small hemorrhagic multiple foci and small foci of chronic inflammation. Immunohistochemical analysis revealed the presence of intracellular $S$. neurona meronts and free merozoites in brain and spinal cord samples. The PCR-RFLP analysis showed a specific $S$. neurona restriction pattern in brain samples. Previous detection of antibodies to $S$. neurona in horses, the abundance of opossums in the region and the specific confirmation in this case, suggest that the disease may be widespread in Argentina. This is the first EPM case report in Argentina, confirmed by histopathologic, immunohistochemical and molecular studies.
\end{abstract}

\section{Key words}

Sarcocystis neurona, EPM, diagnosis, Argentina

Fecha de recepción: 27/02/2019

Fecha de revisión: 04/06/2019

Fecha de aprobación: 05/06/2019
ANALECTA Vet 2019; Enero-Junio; 39(1):32-36

Impresa ISSN 03655 14-8 Electrónica ISSN 1514-2590

doi.org10.24215/15142590e035 


\section{Introducción}

La mieloencefalitis equina por protozoos (sigla en inglés EPM = equine protozoal myeloencephalitis) es una enfermedad causada, principalmente, por Sarcocystis neurona, que genera pérdidas económicas significativas en la producción equina (Dubey et al., 2001). Los protozoarios apicomplexa del género Sarcocystis se caracterizan por presentar ciclos indirectos del tipo predador-presa, con formación de quistes musculares en los hospedadores intermediarios y multiplicación sexual y producción de esporocistos infectantes en el intestino de los hospedadores definitivos (Dubey et al., 2001). La distribución de EPM causada por S. neurona está restringida al continente americano, siguiendo la distribución de los huéspedes definitivos, las comadrejas Didelphis albiventris y Didelphis virginiana (Dubey et al., 2000; Fenger et al., 1995). Neospora caninum y Neospora hughesi son otros protozoarios formadores de quistes tisulares, reportados ocasionalmente como agentes causantes de EPM (Dubey et al., 2001; Hamir et al., 1998).

La EPM se caracteriza por presentar signos neurológicos relacionados con lesiones multifocales del sistema nervioso central, siendo la atrofia muscular y las dificultades en la marcha los signos más relevantes. Los métodos serológicos (principalmente inmunoblot y ELISA) son los recomendados para el diagnóstico in vivo, mientras que la histopatología, la inmunohistoquímica (IHQ) y la reacción en cadena de la polimerasa seguida de análisis de fragmentos de restricción (PCR-RFLP) son algunos de los métodos recomendados para el diagnóstico post mortem (Dubey et al., 2001; Tanhauser et al., 1999; Yeargan \& Howe 2011). En Argentina, se detectaron anticuerpos contra $S$. neurona en el $26 \%$ de 640 equinos muestreados, observándose una seroprevalencia mayor en equinos con signos neurológicos que en animales asintomáticos (39,2 \% versus $22,1 \%)$; sin embargo, no se han descripto y confirmado casos clínicos mediante estudios post mortem (Moré et al., 2014). En estudios realizados sobre muestras de equinos asintomáticos, no se han detectado anticuerpos contra especies de Neospora o se detectaron en niveles bajos (Dubey et al., 1999; Rojas et al., 2016).

La comadreja overa (D. albiventris) tiene una amplia distribución en la mayoría de las zonas rurales y áreas suburbanas de nuestro país. Sin embargo, los intentos de aislar $S$. neurona de materia fecal o intestino de comadrejas de Argentina dieron como resultado la identificación de otras especies de Sarcocystis (Dubey et al., 2000; Dubey et al., 1999).

El objetivo de esta comunicación fue describir un caso de EPM causado por $S$. neurona en un equino argentino, confirmado mediante métodos histopatológicos, inmunohistoquímicos y moleculares.

\section{Presentación del caso}

Una yegua de 12 años, cruza, nacida y criada en un haras del partido de Cañuelas, provincia de Buenos Aires, Argentina, presentó marcha asincrónica progresiva, por lo que los médicos veterinarios del establecimiento instauraron un tratamiento sintomático con fenilbutazona (o,1 mg/Kg) y $3 \mathrm{~g} /$ día de vitamina B1, durante 15 días, sin resultados positivos. Posteriormente, la yegua mostró arpeo bilateral, dismetría e inestabilidad en la marcha, por lo que se decidió administrar la droga anticonvulsiva fenitoína (15 g/día) durante 75 días. También se efectuó la tenectomía del músculo extensor digital lateral de los miembros pelvianos, como tratamiento paliativo ante la sospecha de una intoxicación por ingestión de achicoria amarilla o diente de león (Hypochoeris radicata) (Torre, 2005), lo que produjo una mejoría leve en la marcha. A pesar del tratamiento, el cuadro clínico fue progresivo con depresión, respuesta deficiente a los estímulos, parálisis facial (ptosis auricular, palpebral, nasal y labial) y atrofia del músculo masetero. Además, presentó atrofia grave de los músculos del dorso, lomo y miembros pelvianos, temblores generalizados, arrastre severo de los cascos y tetraparesia. Se formuló un diagnóstico clínico de mieloencefalitis multifocal. Debido a la severidad e irreversibilidad del cuadro clínico y a la falta de respuesta a los tratamientos, se decidió la eutanasia (sedación: acepromacina o,05 $\mathrm{mg} / \mathrm{kpv}$ y xilacina $1,1 \mathrm{mg} / \mathrm{kpv}$; anestesia: ketamina $3,5 \mathrm{mg} / \mathrm{kpv}$ y por último $100 \mathrm{ml}$ de clorhidrato de procaína al $60 \%$ endovenoso rápido), cinco meses después del inicio de los signos clínicos.

En la necropsia no se observaron lesiones en el sistema nervioso central ni en otros órganos. Se recolectaron muestras representativas de cerebro y médula espinal y se fijaron en formol tamponado al $10 \%$ para estudios histopatológicos y se congelaron a $-20{ }^{\circ} \mathrm{C}$ para estudios moleculares.

Las muestras para histopatología se procesaron mediante técnicas de rutina y las secciones de tejido se tiñeron con hematoxilina y eosina. Las lesiones, en el tálamo y el metencéfalo, consistieron en múltiples y pequeños focos hemorrágicos y pequeños focos de inflamación crónica conformados, principalmente, por histiocitos y ocasionales células multinucleadas y células espumosas (gitter cells). En la médula espinal se observaron necrosis neuronal, satelitosis, infiltrados mononucleares perivasculares, tumefacción endotelial y áreas dispersas de degeneración axonal.

La reacción de inmunohistoquímica se llevó a cabo con una técnica comercial basada en la unión de estreptavidina y biotina (LSAB, Dako Cytomation, Carpinteria, USA) utilizando un suero policlonal anti-S. neurona producido en conejo como anticuerpo primario, proporcionado, gentilmente, por el Dr. J. P. Dubey (Animal Parasitic Disease Laboratory, U.S. Department of 
Agriculture, Beltsville, USA). Mediante este estudio se reveló la presencia de merontes intracelulares de $S$. neurona y merozoitos libres en muestras de cerebro y médula espinal (Fig. 1).

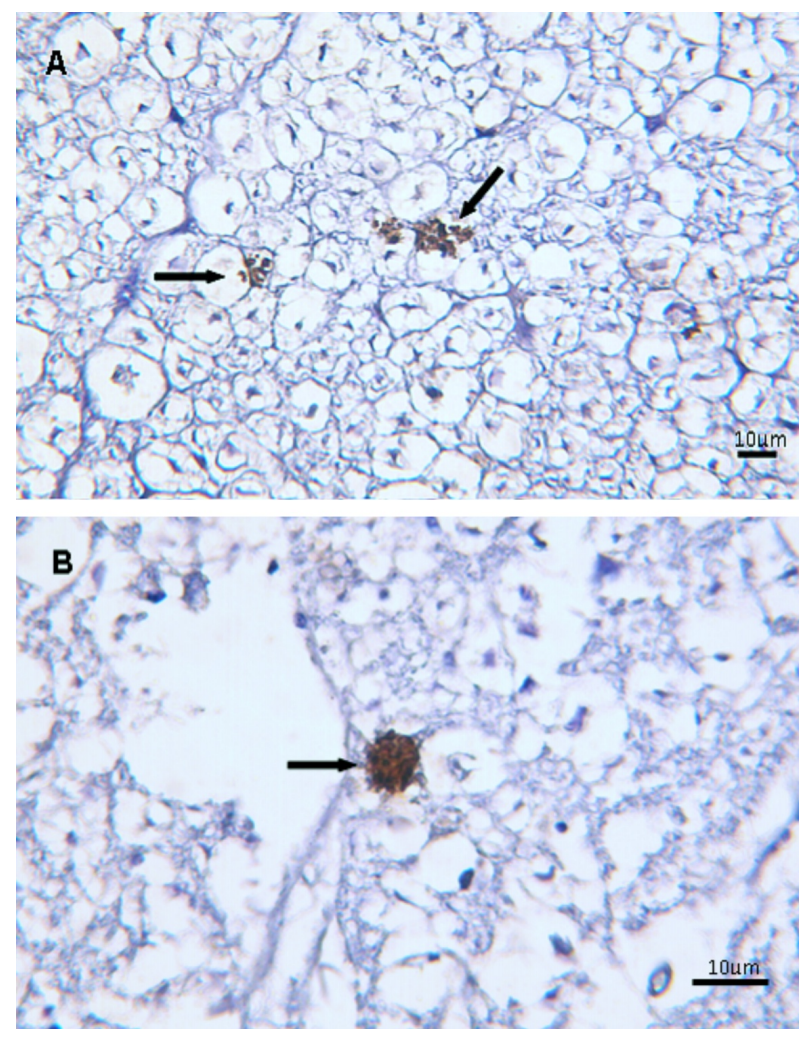

Figura 1. Fotomicrografías que muestran el resultado positivo de la inmunohistoquímica (LSAB, Dako Cytomation) para $S$. neurona en muestras de médula espinal (A) y cerebro (B). Se marcan específicamente (color pardo) merozoitos y merontes de $S$. neurona (flechas).

El ADN se extrajo a partir de $50 \mathrm{mg}$ de cada tejido congelado utilizando un kit comercial (Wizard® Genomic DNA Purification Kit, Promega), procediendo de acuerdo con las instrucciones del fabricante e incubando la digestión con proteinasa $\mathrm{K}$ a $55^{\circ} \mathrm{C}$ durante toda la noche. Se amplificó el ADN mediante dos técnicas de PCR y se procedió al corte del amplificado con enzimas de restricción acorde protocolos descriptos previamente (Tanhauser et al., 1999). Se observó un patrón de restricción específico de $S$. neurona en muestras de cerebro mediante PCR-RFLP utilizando los primers JNB33-JNB54 digeridos con HinfI y DraI $\left(37^{\circ} \mathrm{C}, 1 \mathrm{~h}\right)$, y los primers JNB25-JD396 digeridos con HinfI y HindIII $\left(37^{\circ} \mathrm{C}, 1 \mathrm{~h} 30 \mathrm{~min}\right)$ (Fig. 2). El ADN del control positivo fue proporcionado gentilmente por el Dr. D. Howe (Department of Veterinary Science, Gluck Equine Research Center, University of Kentucky, USA).

Sobre la base de todos los resultados antes expuestos, se formuló un diagnóstico de mieloencefalitis producida por Sarcocystis neurona.

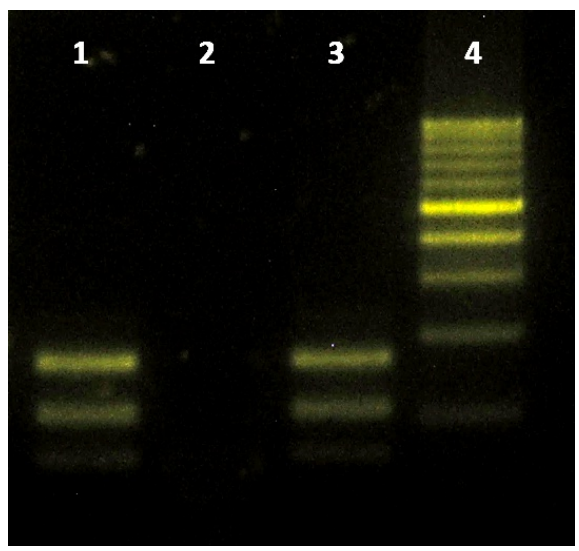

Figura 2. Patrón de restricción del fragmento amplificado mediante los primers JNB25-JD396 mediante la enzima HinfI. Electroforesis en gel de agarosa al 1,5 \%, tinción con SYBRsafe y observación en transiluminador de luz azul (Invitrogen, USA). Referencias: $1=$ SNC del equino; $2=$ control negativo; $3=\mathrm{ADN}$ S. neurona (control positivo); 4= Marcador de peso molecular, Cienmarker (Biodymamics, Argentina).

\section{Discusión y conclusiones}

La presente comunicación describe un caso clínico con una progresión de los signos y con lesiones similares a las informadas previamente en otros casos de EPM (Dubey et al., 2001). Además, se confirmó la presencia de $S$. neurona en el tejido nervioso afectado mediante métodos inmunohistoquímicos y moleculares. Los tratamientos aplicados por los veterinarios actuantes en este caso fueron los usuales en el manejo general de los trastornos neurológicos, pero no mostraron ningún efecto sobre la infección por $S$. neurona. Mackay (2006) informó que el tratamiento específico con sulfadiazina-pirimetamina o ponazuril podría producir una mejoría en el $60 \%$ de los casos. Por lo tanto, la aplicación de estos medicamentos podría tenerse en cuenta para tratar las etapas tempranas de trastornos neurológicos en casos similares. Estos medicamentos también podrían ayudar en la orientación del diagnóstico de EPM basado en la respuesta al tratamiento (Dubey et al., 2001).

La falta de lesiones macroscópicas en el presente caso podría deberse a una etapa crónica de EPM, lo que estaría de acuerdo con el curso clínico y confirmado por el estudio histopatológico. La mayoría de los casos de EPM reportados se corresponden con enfermedad aguda, mostrando hemorragia severa y lesiones principalmente en la médula espinal y en las meninges (Boy et al., 1990; Dubey et al., 2001). En el presente estudio, las lesiones microscópicas consistieron en pequeños y múltiples focos de hemorragias e inflamación crónica en el tálamo, el metencéfalo y la médula espinal. Además, la presencia de $S$. neurona en estos tejidos se identificó mediante inmunohistoquímica y PCR-RFLP, lo que se indica 
para confirmar la etiología de EPM (Boy et al., 1990; Dubey et al., 2001; Hamir et al., 1993). Lamentablemente, no se conservaron muestras de suero de este animal para realizar estudios serológicos que complementaran el diagnóstico.

Diferentes cepas o aislamientos de $S$. neurona evidencian diferentes antígenos de superficie que podrían ser causas de diferencias en el desarrollo de los casos clínicos, como así también influir en el diagnóstico serológico y molecular (Howe et al., 2008). Teniendo esto en cuenta, se llevará a cabo una caracterización molecular adicional de $S$. neurona en las muestras de este caso, así como de otras que contengan $\mathrm{ADN}$ de $S$. neurona, para identificar las variantes genotípicas circulantes y ampliar la comprensión de aspectos epidemiológicos de la EPM en Argentina.

La detección previa de anticuerpos contra $S$. neurona en caballos, la abundancia de comadrejas (Didelphis albiventris) en el medio y la confirmación específica en este caso, sugieren que la enfermedad puede estar muy extendida en Argentina. Esta es la primera descripción de un caso de EPM en Argentina, confirmado mediante estudios histopatológicos, inmunohistoquímicos y moleculares. La confirmación del diagnóstico de esta enfermedad en nuestro medio sirve de alerta a los veterinarios para que incluyan a la EPM entre los diagnósticos diferenciales ante trastornos neurológicos en equinos.

\section{Agradecimientos}

Los autores agradecen al Dr. J. P. Dubey y al Dr. D. Howe por proporcionar el anticuerpo primario anti $S$. neurona y el ADN control de $S$. neurona, respectivamente.

\section{Conflicto de intereses}

Los autores declaran que no existe conflicto de intereses, incluyendo entre estos últimos las relaciones financieras, personales o de otro tipo con otras personas u organizaciones que pudieran influir de manera inapropiada en el trabajo.

\section{Bibliografía}

Boy MG, Galligan DT, Divers TJ. 1990. Protozoal encephalomyelitis in horses: 82 cases (1972-1986). Journal of the American Veterinary Medical Association. 196:632-4.

Dubey JP, Lindsay DS, Saville WJ, Reed SM, Granstrom DE, Speer CA. 2001. A review of Sarcocystis neurona and equine protozoal myeloencephalitis (EPM). Veterinary Parasitology. 95:89-131.

doi: 10.1016/So304-4017(oo)oo384-8
Dubey JP, Speer CA, Bowman DD, Horton KM, Venturini MC, Venturini L. 2000. Experimental transmission of Sarcocystis speeri Dubey and Lindsay, 1999 from the South American opossum (Didelphis albiventris) to the North American opossum (Didelphis virginiana). Journal of Parasitology. 86:624-7. doi: 10.1645/00223395(2000)o86[0624:ETOSSD]2.0.CO;2

Dubey JP, Venturini L, Venturini C, Basso W, Unzaga J. 1999. Isolation of Sarcocystis falcatula from the South American opossum (Didelphis albiventris) from Argentina. Veterinary Parasitology. 86:239-44.

doi: 10.1016/So304-4017(99)oo145-4

Dubey JP, Venturini MC, Venturini L, McKinney J, Pecoraro M. 1999. Prevalence of antibodies to Sarcocystis neurona, Toxoplasma gondii and Neospora caninum in horses from Argentina. Veterinary Parasitology. 86:59-62.

doi: 10.1016/So304-4017(99)00127-2

Fenger CK, Granstrom DE, Langemeier JL, Stamper S, Donahue JM, Patterson JS, Gajadhar AA, Marteniuk JV, Xiaomin Z, Dubey JP. 1995. Identification of opossums (Didelphis virginiana) as the putative definitive host of Sarcocystis neurona. Journal of Parasitology. 81:916-9.

doi: $10.2307 / 3284040$

Hamir AN, Moser G, Galligan DT, Davis SW, Granstrom DE, Dubey JP. 1993. Immunohistochemical study to demonstrate Sarcocystis neurona in equine protozoal myeloencephalitis. Journal of Veterinary Diagnostic Investigation. 5:418-22.

doi: 10.1177/104063879300500320

Hamir AN, Tornquist SJ, Gerros TC, Topper MJ, Dubey JP. 1998. Neospora caninum-associated equine protozoal myeloencephalitis. Veterinary Parasitology. 79:269-74.

doi: 10.1016/So304-4017(98)oo178-2

Howe DK, Gaji RY, Marsh AE, Patil BA, Saville WJ, Lindsay DS, Dubey JP, Granstrom DE. 2008. Strains of Sarcocystis neurona exhibit differences in their surface antigens, including the absence of the major surface antigen SnSAG1. International Journal for Parasitology. 38:623-31.

doi: 10.1016/j.ijpara.2007.09.007

Mackay R. 2006. Equine protozoal myeloencephalitis: treatment, prognosis, and prevention. clinical techniques in equine practice. 5:9-16.

doi: 10.1053/j.ctep.2006.01.003

Moré G, Vissani A, Pardini L, Monina M, Muriel M, Howe D, Barrandeguy M, Venturini MC. 2014. Seroprevalence of Sarcocystis neurona and its association with neurological disorders in Argentinean horses. Journal of Equine Veterinary 
Science. 34:1051-4.

doi: 10.1016/j.jevs.2014.06.002

Rojas MC, Moré G, Campero LM, Fort M, Giménez H, Venturini MC. 2016. Detection of Neospora spp. antibodies in horses from La Pampa, Argentina. 10th International Equine Infectious Diseases Conference (IEIDC X). Buenos Aires, Argentina. Journal of Equine Veterinary Science 39: S50. doi: 10.1016/j.jevs.2016.02.110

Tanhauser SM, Yowell CA, Cutler TJ, Greiner EC, Mackay RJ, Dame JB. 1999. Multiple DNA markers differentiate Sarcocystis neurona and
Sarcocystis falcatula. The Journal of Parasitology. 85:221-8. doi: 10.2307/3285623

Torre F. 2005. Clinical diagnosis and results of surgical treatment of 13 cases of acquired bilateral stringhalt (1991-2003). Equine Veterinary Journal. 37:181-3.

doi: 10.2746/0425164054223877

Yeargan MR, Howe DK. 2011. Improved detection of equine antibodies against Sarcocystis neurona using polyvalent ELISAs based on the parasite SnSAG surface antigens. Veterinary Parasitology. 176:16-22. doi: 10.1016/j.vetpar.2010.10.034 